\title{
及2-adrenergic agonists and doping: Where do we stand?
}

To the Editor,

Hostrup et $\mathrm{al}^{1}$ kindly commented on our previous paper related to the enhancement of physical performance by $\beta 2$-agonists. ${ }^{2}$ First, we would like to thank the authors for their review and acknowledge that our title and conclusions may have been misleading because, in fact, they only refer to endurance/aerobic-dominated performances. However, we were very clear that our results were based on VO2max and time to exhaustion at this intensity. We further stressed that VO2max measures do not represent a direct measure of physical performance, although $\mathrm{VO} 2 \mathrm{max}$ is considered a variable of physical fitness and training status of each individual. We agree that we could have warned of the fact that this article only analyses efforts mainly aerobic, but, knowing that the attentive reader would check our inclusion and exclusion criteria, we chose to alert to other facts not so clear throughout the text, of the results and annexes.

In our meta-analysis, only the study by Koch et al ${ }^{3}$ favoured $\beta 2$ agonists (salbutamol) over placebo, when comparing VO2max, but this study included only female athletes. No other studies, which included mixed-gender populations or only male athletes, showed improved exercise performance. When evaluating time to exhaustion, one study by Collomp et al ${ }^{4}$ favoured $\beta 2$-agonists over placebo. However, these authors failed to reproduce these results in similar circumstances. ${ }^{5}$

Following our findings, a recent systematic review and metaanalysis by Riiser et $\mathrm{al}^{6}$ showed that $\beta 2$-agonists do not affect maximal oxygen consumption in non-asthmatic subjects regardless of type, dose, administration route, duration of treatment or performance level of participants. In addition, $\beta 2$-agonists did not improve aerobic physical performance measured by close-ended or openended tests.

Likewise, we agree with Hostrup et al that there is evidence that short efforts/anaerobic-dominated performances may be improved by $\beta 2$-agonists, ${ }^{7}$ and that $\beta 2$-agonists exert anabolic and lipolytic actions $^{8}$; although it is unclear if those actions might be obtained with currently permitted drugs, dose and administration route. However, we must also take into account that, like our study, this same review that the authors point out to support improved performance in anaerobic and short-term efforts has limitations mentioned by the authors themselves. For example, the included studies evaluated efforts that are often not representative of performance in the studied exercise activities, with individuals without experience, occasional practitioners (non-competitive level participants; untrained), and that was notorious in the heterogeneity of results in the variables presented. $^{7}$ Moreover, characteristic side effects of $\beta 2$-agonists (tremor, tachycardia) may break the blinding in aware participants affecting the results.

Furthermore, $\beta 2$-agonists are subjected to in and out of competition controls, which may hinder its use as leaning and ergogenic agents outside competition. The additional control of masking agents may also impair said use, as will the tolerance to bronchoprotective and bronchodilator effects by the regular use of inhaled $\beta$-agonists, and the persistence of side effects in some susceptible asthmatics.

While regular inhaled therapeutic use of $\beta 2$-agonists such as salbutamol, formoterol and salmeterol is permitted under the current World Anti-Doping Agency (WADA) regulations; for the treatment of exacerbations, higher doses or other routes of administration may be needed. In addition, most athletes with asthma will need daily inhaled corticosteroid maintenance therapy or other drugs to avoid potential side effects or reduced tolerance and efficacy of short-acting $\beta 2$ agonists. Likewise, given the pharmacokinetic individual differences, some athletes might exceed the permitted urine threshold for salbutamol or formoterol, when administering inhaled doses within limits. So, it is advisable for professional athletes with asthma and/or bronchial hyperreactivity to apply for a therapeutic use exemption (TUE). ${ }^{9}$

As our article was proposed to be written as a letter to the editor, the authors had a series of limitations (namely concerning the number of words) that conditioned the discussion of some topics, such as these proposed by Hostrup.

\section{CONFLICT OF INTEREST}

The authors have no conflict of interest to declare.

\section{FUNDING INFORMATION}

No funding sources for the present paper.

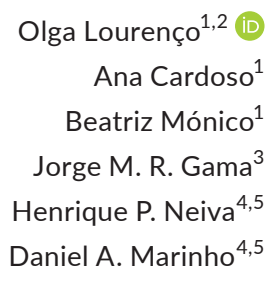

${ }^{1}$ Faculty of Health Sciences, University of Beira Interior, Covilhã, Portugal

${ }^{2}$ CICS-UBI Health Sciences Research Centre, University of Beira Interior, Covilhã, Portugal 
${ }^{3}$ Centre of Mathematics and Applications (CMA-UBI), University of Beira Interior, Covilhã, Portugal

${ }^{4}$ Department of Sport Sciences, University of Beira Interior,

Covilhã, Portugal

${ }^{5}$ Research Center in Sports Sciences, Health Sciences and Human Development, CIDESD, Covilhã, Portugal

\section{Correspondence}

Olga Lourenço, CICS-UBI Health Sciences Research Centre, Avenida Infante D. Henrique, 6200-506 Covilhã, Portugal.

Email: olga@fcsaude.ubi.pt

\section{ORCID}

Olga Lourenço (1) https://orcid.org/0000-0002-8401-5976

\section{REFERENCES}

1. Hostrup M, Jessen S, Backer V, Bangsbo J, Jacobson GA. Beta2 -adrenergic agonists can enhance intense performance and muscle strength in healthy individuals. Allergy. 2021. https://doi. org/10.1111/all.14735

2. Cardoso A, Monico B, Gama JMR, Neiva HP, Marinho DA, Lourenco O. beta2-adrenergic agonists do not improve physical performance in healthy individuals. Allergy. 2020. https://doi.org/10.1111/ all.14588
3. Koch S, Karacabeyli D, Galts C, Maclnnis MJ, Sporer BC, Koehle MS. Effects of inhaled bronchodilators on lung function and cycling performance in female athletes with and without exercise-induced bronchoconstriction. J Sci Med Sport. 2015;18(5):607-612.

4. Collomp K, Candau R, Lasne F, Labsy Z, Prefaut C, De Ceaurriz J. Effects of short-term oral salbutamol administration on exercise endurance and metabolism. J Appl Physiol (1985). 2000;89(2):430-436.

5. Collomp K, Candau R, Millet G, et al. Effects of salbutamol and caffeine ingestion on exercise metabolism and performance. Int $J$ Sports Med. 2002;23(8):549-554.

6. Riiser A, Stensrud T, Stang J, Andersen LB. Aerobic performance among healthy (non-asthmatic) adults using beta2-agonists: a systematic review and meta-analysis of randomised controlled trials. $\mathrm{Br}$ J Sports Med. 2020. https://doi.org/10.1136/bjsports-2019-100984

7. Riiser A, Stensrud T, Stang J, Andersen LB. Can beta2-agonists have an ergogenic effect on strength, sprint or power performance? Systematic review and meta-analysis of RCTs. Br J Sports Med. 2020;54(22):1351-1359.

8. Hostrup M, Jacobson GA, Jessen S, Lemminger AK. Anabolic and lipolytic actions of beta2 -agonists in humans and antidoping challenges. Drug Test Anal. 2020;12(5):597-609.

9. Allen H, Backhouse SH, Hull JH, Price OJ. Anti-doping Policy, therapeutic use exemption and medication use in athletes with asthma: a narrative review and critical appraisal of current regulations. Sports Med. 2019;49(5):659-668. 